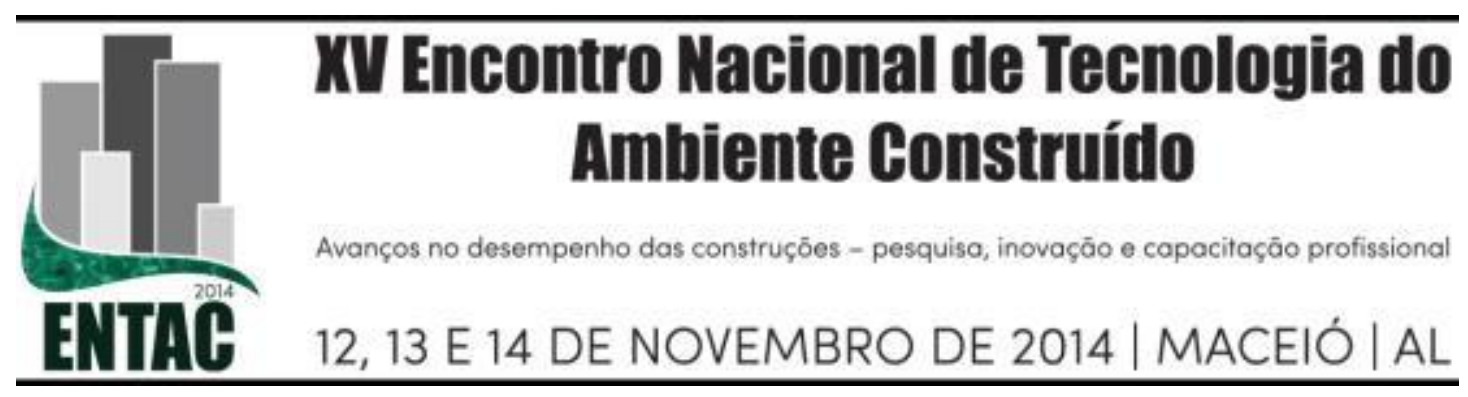

\title{
CERTIFICAÇÕES HABITACIONAIS E A AVALIAÇÃO DO CICLO DE VIDA
}

\author{
CARDOSO, Poliana F. (1); PABLOS, Javier. M. (2)
}

(1) Instituto de Arquitetura e Urbanismo, Universidade de São Paulo, e-mail: polistm@usp.sc.br; (2) Instituto de Arquitetura e Urbanismo, Universidade de São Paulo, e-mail: pablos@sc.usp.br

\begin{abstract}
RESUMO
Para que haja melhor desempenho na integração das tomadas de decisões no processo de projeto e construção, a certificação entra como auxílio em direção a sustentabilidade. Neste contexto, o trabalho tem como objetivo discutir a aplicação da técnica de ACV (Avaliação do Ciclo de Vida) em sistemas de CAE (Certificação Ambiental de Edificações) habitacional no cenário brasileiro nas fases iniciais de projeto do ambiente construído. Para alcançar este objetivo utiliza-se a pesquisa exploratória por meio das etapas metodológicas: a) revisão bibliográfica sobre desempenho ambiental, certificação ambiental de edificações e a técnica de $\mathrm{ACV}$; b) seleção dos sistemas para discussão; c) análise em relação às principais características de cada sistema, categorias de impacto com abordagem de ACV, fases de ciclo de vida da edificação utilizadas e, correlaciona os aspectos e impactos dos principais métodos de caracterização da fase de AICV (Avaliação de Impacto do Ciclo de Vida) com as categorias de impacto dos sistemas selecionados; d) discussão dos resultados destas análises. Os resultados mostram que as CAE utilizam a ACV de forma incipiente para certificar em alguns critérios, como, materiais, resíduos e energia. Outro resultado é a utilização dos métodos de caracterização nas certificações, as CAE utilizam categorias de avaliação de impacto parecidas com as categorias do método EPS (Environmental Priority Strategies) e menos com dos métodos ECO-Indicator e EDIP (Environmental Design for Industrial Products). Deste modo, para facilitar a utilização da ACV em certificações é necessário que os critérios das certificações sejam além de classificações por critérios de categorias e tenham banco de dados público dos produtos a serem utilizados na edificação.
\end{abstract}

Palavras-chave: Avaliação do Ciclo de Vida, Sistemas de Certificação Ambiental, Desempenho Ambiental de Habitações.

\begin{abstract}
To ensure improved performance in the integration of decision making in the design and construction process, the certification comes as aid towards sustainability. In this context, the paper aims to discuss the technique of LCA (Life Cycle Assessment) in housing CAE systems (Environmental Certification of Building) on the Brazilian scene in the early stages of design of the built environment. To achieve this goal is used the exploratory research through methodological stages: a) Literature review about environmental performance, environmental certification of buildings and technical LCA; b) Selection of systems for discussion; c) Analysis in relation the main characteristics of each system, impact categories with LCA approach, phases of the lifecycle building that are used and correlates aspects and impacts of the main methods of characterization phase LCIA (Impact Assessment Life Cycle) with impact categories of the selected systems; d) discuss of results of these analyzes. The results reveal that CAE use LCA incipiently to certify on some criteria as materials, waste and energy. Another result is the use of methods characterization of certificates, CAE use categories of impact assessment similar to the categories of EPS (Environmental Priority Strategies) and less with the methods ECO-Indicator and EDIP (Environmental Design for Industrial Products) method. Thus, to facilitate the use of LCA in certification is necessary that criteria of certifications be in addition to ratings by criteria categories and has public database of products to be used in building.
\end{abstract}


Keywords: Life Cycle Assessment, Environmental Certification Systems, Environmental Performance of Housing.

\section{INTRODUÇÃO}

A evolução dos conceitos de desenvolvimento sustentável e novos modelos econômicos fez com que o setor da construção civil se posicionasse estrategicamente frente aos impactos por ela causados, introduzindo no mercado sistemas de avaliação ambiental de edificações derivados dos procedimentos de avaliação ambiental de processos ou produtos (SEVERO; CARVALHO FILHO; SOUSA, 2012; SILVA, 2003). Esses sistemas de avaliação são qualitativos, se baseiam no desempenho relativo ambiental de uma construção, quando comparada a outros edifícios de diferentes alternativas de concepção (COLE, 1998).

Estes sistemas surgiram na década de 90 na Europa, EUA e Canadá, com o intuito de medir o desempenho ambiental em prol do desenvolvimento da sustentabilidade. Para o contexto brasileiro estes sistemas foram trazidos de forma generalizada por não apresentarem flexibilidade de adaptação a diferentes contextos locais, como por exemplo, o LEED - Leadership in Energy and Environmental Design, sistema americano (BUENO, 2010), ou BREEAM - Building Research Establishment Environmental Assessment Method, da Inglaterra.

Segundo Rebitzer (2004), para atingir o desenvolvimento sustentável necessita-se de sistemas e ferramentas que ajudem a quantificar e comparar os impactos ambientais dos bens (produtos) e serviços para a sociedade.

A técnica internacionalmente aceita para esta avaliação ambiental de edifícios é a ACV - Avaliação do Ciclo de Vida, do inglês, LCA - Life Cycle Analysis, originalmente definida pela SETAC - Society of Environmental Toxicology and Chemistry, como sendo um processo para avaliar as implicações ambientais de um produto ou atividade em todos os estágios de sua vida que geram impactos (SETAC, 1991) ou seja, permite avaliar os impactos de um sistema de produto, sistematicamente, por todo o seu ciclo de vida. O ciclo de vida de um sistema de produto permeia seus estágios sucessivos e encadeados, desde a extração dos recursos naturais até a disposição final (ABNT, 2009; SILVA, 2007).

Neste contexto, este trabalho tem como objetivo discutir a aplicação da técnica de ACV em sistemas de CAE - Certificação Ambiental de Edificações habitacional no cenário brasileiro nas fases iniciais de projeto do ambiente construído.

\subsection{Desempenho Ambiental - DA}

Define-se como DA os resultados mensuráveis da gestão de uma organização sobre seus aspectos ambientais (ABNT, 2004). Este desempenho foca em categorias que tem relação com o meio exterior (derramamentos no solo, descarte de resíduos e as emissões para a atmosfera) e relação com o meio ambiente interior (ergonomia, segurança, saúde, conforto ambiental, lumínico, acústico e higro-térmico) $\mathrm{O}$ desempenho ambiental da edificação está relacionado a todos os elementos que compõem esta edificação, considerando uma complexa gama de aspectos ambientais (ZAMBRANO; BASTOS; SLAMA, 2004).

\subsection{Certificações Ambientais de Edificações - CAE}

As CAE são sistemas desenvolvidos para Avaliação do Desempenho Ambiental (ADA) da construção em fase de planejamento; construção / reforma; e operação. Podem ser utilizados além da tipologia habitacional, para avaliar condomínios, loteamentos, escolas e unidades comerciais / industriais. Tem o objetivo de fornecer aos especialistas 
sobre o desempenho ambiental das diversas áreas analisadas da construção (ZAMBRANO; BASTOS; SLAMA, 2004).

Em relação à divulgação as CAE podem ser "orientadas para o mercado" ou "orientadas para pesquisa". Para as orientadas ao mercado, as avaliações são desenvolvidas para serem de fácil acesso aos projetistas ou para divulgar a importância da dedicação continua de melhorar a qualidade ambiental de projetos, obra e operação pelo mercado. Para os orientados a pesquisa, as avaliações evidenciam o desenvolvimento de uma metodologia com base científica aprofundada com o intuito de colaborar com novos sistemas (SILVA, 2003).

\subsection{Avaliação do Ciclo de Vida- ACV}

A ACV pode ser usada para avaliar sistemas complexos como as edificações. A avaliação se baseia em quatro fases: (1) definição de objetivo e escopo, (2) análise de inventário (ICV), (3) avaliação de impactos (AICV), e (4) interpretação (ABNT, 2009).

Uma das fases considerada importante para a interpretação dos resultados é a AICV. Nesta fase os dados coletados na fase anterior (ICV) são caracterizados em resultados de impacto de indicadores associados ao sistema de produto em estudo, ampliando o entendimento de sua significância ambiental (ABNT, 2009).

Existem diversos métodos de caracterização desenvolvidos nas últimas décadas que utilizam diferentes categorias de impacto a serem avaliadas, pode-se destacar: Ecoindicator99 (2001), CML2001, EPS2000, LIME (2003), EDIP97 e IMPACT2002+.

Recomenda-se para a avaliação de uma edificação utilizar indicadores para verificar categorias como: (i) consumo de materiais, (ii) consumo de energia, (iii) consumo de água, (iv) geração e liberação de resíduos, (v) uso e ocupação do solo e (vi) qualidade do ar interior (KLEIN, 2002).

\section{MÉTODO DE PESQUISA}

Para esta pesquisa bibliográfica e exploratória foram adotadas as seguintes etapas metodológicas: (i) Seleção dos sistemas para discussão; (ii) Análise em relação às principais características de cada sistema; (iii) Identificação das categorias de impacto com abordagem de ACV; (iv) Identificação das fases do ciclo de vida da edificação utilizadas nos sistemas e (v) Correlação dos aspectos e impactos dos principais métodos de caracterização da fase de AICV (Avaliação de Impacto do Ciclo de Vida) com as categorias de impacto dos sistemas selecionados.

\section{RESULTADOS}

\subsection{Seleção dos sistemas para discussão}

No Brasil existem alguns sistemas de certificação ambiental utilizados de forma nacional ou local. Neste trabalho foram levantados os sistemas mais difundidos e após realizada a seleção para o estudo. Para este estudo foram selecionados os de uso no contexto nacional para edificações habitacionais: AQUA, BREEAM, selo Casa Azul e a certificação LEED. A seleção destes sistemas levou em consideração a disponibilidade de informações bibliográficas, o país, forma de avaliação, categorias e critérios adotados como referência por cada sistema. 


\subsection{Análise em relação às principais características de cada sistema}

Para que os sistemas pudessem ser vistos de um panorama geral foram analisadas características principais em cada um e os resultados são mostrados na Figura1.

Figura 1 - Características principais de cada sistema de certificação ambiental

\begin{tabular}{|c|c|c|c|c|c|}
\hline & \multicolumn{2}{|c|}{ AQUA } & \multirow[b]{2}{*}{\begin{tabular}{l}
\multicolumn{1}{c}{ BREEAM } \\
1- Energia e emissões \\
de CO2 \\
2- Água \\
3- Materiais \\
4- Escoamento das \\
águas da superfície \\
5- Resíduos \\
6- Poluição \\
7- Saúde e bem-estar \\
8- Gestão \\
9- Ecologia
\end{tabular}} & \multirow{2}{*}{\begin{tabular}{|l|}
\multicolumn{1}{|c|}{ CASA AZUL } \\
1-Qualidade Urbana \\
2-Projeto e Conforto \\
3-Eficiência Energética \\
4-Conservação de \\
Recursos materiais \\
5- Gestão da Água \\
6- Práticas Sociais
\end{tabular}} & \multirow[b]{2}{*}{\begin{tabular}{l}
\multicolumn{1}{c}{ LEED } \\
1-Espaço Sustentável \\
2-Uso racional da água \\
3-Energia e Atmosfera \\
4-Materiais e Recursos \\
5-Qualidade Ambiental \\
Interna \\
6-Inovação e Processo \\
do Projeto \\
7-Créditos Regionais
\end{tabular}} \\
\hline $\begin{array}{l}\text { Categorias de } \\
\text { Avaliação }\end{array}$ & $\begin{array}{l}\text { 1-Relação do edifício } \\
\text { com o seu entorno } \\
\text { 2-Qualidade dos } \\
\text { componentes } \\
\text { 3-Canteiro } \\
\text { responsável } \\
\text { 4-Gestão de energia } \\
\text { 5-Gestão de água } \\
\text { 6-Gestão dos } \\
\text { Resíduos } \\
\text { 7-Gestão da } \\
\text { conservação e da } \\
\text { manutenção }\end{array}$ & $\begin{array}{l}\text { 8- Conforto } \\
\text { Higrotérmico } \\
\text { 9- Conforto Acústico } \\
\text { 10- Conforto Visual } \\
\text { 11- Conforto Olfativo } \\
\text { 12- Qualidade dos } \\
\text { espaços } \\
\text { 13- Qualidade } \\
\text { Sanitária do Ar } \\
\text { 14- Qualidade } \\
\text { Sanitária da Água }\end{array}$ & & & \\
\hline Aplicabilidade & \multicolumn{2}{|c|}{ Adaptado para aplicação no contexto brasileiro. } & $\begin{array}{l}\text { Desenvolvido para o } \\
\text { Reino Unido, com } \\
\text { aplicação internacional. }\end{array}$ & $\begin{array}{l}\text { Desenvolvido para } \\
\text { aplicação no contexto } \\
\text { brasileiro. }\end{array}$ & $\begin{array}{l}\text { Desenvolvido para o } \\
\text { EUA, com aplicação } \\
\text { internacional. }\end{array}$ \\
\hline $\begin{array}{l}\text { Metodologia de } \\
\text { avaliação }\end{array}$ & \multicolumn{2}{|c|}{$\begin{array}{l}\text { A avaliação dá-se de maneira evolutiva ao longo } \\
\text { da estrutura em árvore composta de Categorias, } \\
\text { Subcategorias e Preocupações. }\end{array}$} & $\begin{array}{c}\text { Avaliação sistemática, o } \\
\text { número total de créditos } \\
\text { ganho para cada área de } \\
\text { critérios das categorias } \\
\text { é multiplicado por um } \\
\text { fator de peso ambiental, } \\
\text { que cada uma tem nas } \\
\text { diferentes regiōes do } \\
\text { mundo. }\end{array}$ & $\begin{array}{c}\text { Avaliação dos edifícios } \\
\text { através de uma lista de } \\
\text { pré-requisitos (checklist) } \\
\text { aos quais são atribuídos } \\
\text { créditos baseados em } \\
\text { uma lista de objetivos } \\
\text { preexistentes. A } \\
\text { classificação final é } \\
\text { obtida pela soma dos } \\
\text { pontos atingidos nas } \\
\text { categorias. }\end{array}$ & $\begin{array}{c}\text { Avaliação dos edificios } \\
\text { através de uma lista de } \\
\text { pré-requisitos (checklist) } \\
\text { aos quais são atribuidos } \\
\text { créditos baseados em } \\
\text { uma lista de objetivos } \\
\text { preexistentes. A } \\
\text { classificação final é } \\
\text { obtida pela soma dos } \\
\text { pontos atingidos nas } \\
\text { categorias. }\end{array}$ \\
\hline $\begin{array}{l}\text { Complexidade de } \\
\text { aplicação }\end{array}$ & \multicolumn{2}{|c|}{$\begin{array}{l}\text { Aplicação simples, no formato de questionário, } \\
\text { aplicado por consultoria. }\end{array}$} & $\begin{array}{l}\text { Aplicação simples, em } \\
\text { forma de questionário, } \\
\text { aplicado por consultoria. }\end{array}$ & $\begin{array}{l}\text { Aplicação simples, no } \\
\text { formato de checklist, de } \\
\text { fácil preenchimento. }\end{array}$ & $\begin{array}{l}\text { Aplicação simples, no } \\
\text { formato de checklist, de } \\
\text { fácil preenchimento. }\end{array}$ \\
\hline Classificação & \multicolumn{2}{|c|}{\begin{tabular}{|c|} 
Niveis de desempenho: Base (B); Boas Práticas \\
(BP); Melhores Práticas (MP). \\
Nivel global: Passa - 14 categorias em nível B; \\
Bom - entre 1 a 4 estrelas em nível BP e MP; \\
Muito Bom - entre 5 a 8 estrelas em nivel BP e \\
MP; Excelente - entre 9 a 11 estrelas em nível \\
BP e MP; Excepcional - com 12 estrelas ou mais.
\end{tabular}} & $\begin{array}{c}\text { Aprovado - } 30 \% \text {; } \\
\text { Bom - } 45 \% ; \\
\text { Muito Bom - 55\%; } \\
\text { Excelente - 70\%; } \\
\text { Excepcional - 85\%. }\end{array}$ & $\begin{array}{l}\text { Bronze - } 19 \text { critérios; } \\
\text { Prata - obrigatórios + } 06 \\
\text { critérios de livre } \\
\text { escolha; } \\
\text { Ouro - obrigatórios + } 12 \\
\text { critérios de livre } \\
\text { escolha. }\end{array}$ & $\begin{array}{c}\text { Certificado }-40-49 \\
\text { pontos } \\
\text { Prata }-50-59 \text { pontos } \\
\text { Ouro }-60-79 \text { pontos } \\
\text { Platina }-80 \text { pontos e } \\
\text { acima. }\end{array}$ \\
\hline
\end{tabular}

Fonte: Autores

\subsection{Identificação das categorias de impacto com abordagem de ACV}

Identifica as categorias de impacto utilizadas pelos sistemas que abordam critérios da ACV para certificar. Através da análise aprofundada de cada critério das categorias de impacto de cada certificação selecionada foi possível identificar estas categorias.

\section{AQUA}

As categorias identificadas no sistema AQUA foram a "Qualidade dos componentes" e “Canteiro responsável”, conforme o Quadro 1.

\section{Quadro 1 - Categorias com abordagem de ACV do AQUA}

\begin{tabular}{|c|c|}
\hline Critério & Descrição \\
\hline \multicolumn{2}{|c|}{ Categoria - Qualidade dos componentes } \\
\hline $\begin{array}{c}\text { Qualidade técnica dos } \\
\text { materiais e equipamentos } \\
\text { utilizados }\end{array}$ & $\begin{array}{c}\text { Neste critério a escolha de produtos e equipamentos apropriados a } \\
\text { edificação e que estejam em conformidade com o Programa Setorial da } \\
\text { Qualidade (PSQ) correspondente a seu âmbito de atuação no programa } \\
\text { Sistema de Qualificação de Materiais, Componentes e Sistemas } \\
\text { Construtivos (SiMaC) do PBQP-H. }\end{array}$ \\
\hline $\begin{array}{c}\text { Qualidade ambiental dos } \\
\text { materiais, produtos e } \\
\text { equipamentos utilizados }\end{array}$ & $\begin{array}{c}\text { As empresas fornecedoras devem fornecer informações referentes a } \\
\text { seus impactos ambientais seja por meio de Fichas de Informação de } \\
\text { Produto ou pelas EPDs (Declarações Ambientais de produto) }\end{array}$ \\
\hline
\end{tabular}




\section{Categoria - Canteiro responsável}

Gestão dos resíduos de $\quad$ Visa realizar um Plano de Gerenciamento de Resíduos da Demolição / \begin{tabular}{l|l} 
canteiro & Desconstrução das edificações.
\end{tabular}

Fonte: FCAV (2013)

\section{BREEAM}

Categorias com enfoque de ACV são Energia e emissões de CO2; Materiais, Resíduos; Saúde e bem-estar; e Gestão e ecologia.

\section{Quadro 2 - Categorias com abordagem de ACV do BREEAM}

\begin{tabular}{|c|c|}
\hline Critério & Descrição \\
\hline \multicolumn{2}{|r|}{ Categoria - Energia e Emiussões de CO2 } \\
\hline Ciclo de armazenamento & Visa tecnologias que emitam baixo carbono ou zero (LZC). \\
\hline \multicolumn{2}{|r|}{ Categoria - Materiais } \\
\hline $\begin{array}{l}\text { Impacto ambiental dos } \\
\text { materiais }\end{array}$ & $\begin{array}{l}\text { Visa a escolha de materiais provenientes de processos de produção } \\
\text { responsáveis. Os materiais são classificados de acordo com um guia } \\
\text { baseado na Avaliação do Ciclo de Vida utilizando a metodologia do } \\
\text { perfil ambiental do material. }\end{array}$ \\
\hline \multicolumn{2}{|r|}{ Categoria - Resíduos } \\
\hline $\begin{array}{l}\text { Armazenamento de } \\
\text { resíduos não recicláveis e } \\
\text { resíduos domésticos } \\
\text { recicláveis }\end{array}$ & $\begin{array}{l}\text { Os materiais recicláveis devem ser discriminados pelo menos três dos } \\
\text { tipos recicláveis (papel, papelão, vidro, plásticos, metais e têxteis). }\end{array}$ \\
\hline $\begin{array}{l}\text { Gestão de resíduos de } \\
\text { construção e a } \\
\text { compostagem }\end{array}$ & $\begin{array}{l}\text { Visa gerenciar a compostagem doméstica e a finalidade dos resíduos da } \\
\text { fase de obra. }\end{array}$ \\
\hline \multicolumn{2}{|r|}{ Categoria - Saúde e Bem-estar } \\
\hline Vida útil da habitação & $\begin{array}{l}\text { Promover a manutenção pra preservar a edificação até a vida útil } \\
\text { prevista em projeto. }\end{array}$ \\
\hline \multicolumn{2}{|r|}{ Categoria - Gestão } \\
\hline $\begin{array}{l}\text { Impactos da construção } \\
\text { local }\end{array}$ & $\begin{array}{l}\text { Visa promover a eficiência dos recursos por meio da gestão eficaz e } \\
\text { adequada dos resíduos do canteiro de obras. }\end{array}$ \\
\hline \multicolumn{2}{|r|}{ Categoria - Ecologia } \\
\hline Valor ecológico do local & Visa acréscimo em terras que o valor para vida selvagem é limitado. \\
\hline Valorização ecológica & $\begin{array}{l}\text { Visa melhoria do local com a plantação de espécies vegetais } \\
\text { recomendadas por ecologistas especialistas. }\end{array}$ \\
\hline $\begin{array}{c}\text { Proteção das } \\
\text { características ecológicas }\end{array}$ & $\begin{array}{l}\text { Visa preservar as áreas de valor ecológico durante a construção e uso } \\
\text { do empreendimento. }\end{array}$ \\
\hline $\begin{array}{l}\text { Mudança nos valores } \\
\text { ecológicos do local }\end{array}$ & $\begin{array}{l}\text { Visa medir o antes e o depois do valor das espécies ecológicas no } \\
\text { desenvolvimento da obra. }\end{array}$ \\
\hline Pegada do edifício & $\begin{array}{c}\text { Visa promover uso eficiente da pegada do edifício, garantindo que o } \\
\text { uso da terra e de materiais seja otimizado por meio da relação da área } \\
\text { útil interna com a área de maior piso. }\end{array}$ \\
\hline
\end{tabular}

Fonte: BRE (2009)

\section{Casa Azul}

Categorias com abordagem de ACV são Projeto e conforto; Conservação de recursos materiais e Práticas sociais. 


\section{Quadro 3 - Categorias com abordagem de ACV do Casa Azul}

\begin{tabular}{|c|c|}
\hline Critério & Descrição \\
\hline \multicolumn{2}{|r|}{ Categoria - Projeto e Conforto } \\
\hline Flexibilidade de projeto & $\begin{array}{l}\text { Solicita que os projetos sejam de fácil adaptação a mudanças no futuro, } \\
\text { como ampliações e modificações, com o intuito de diminuir o consumo, } \\
\text { desperdício de materiais, e menor quantidade de Resíduos de Construção } \\
\text { e Demolição (RCD) e assim aumentar a vida útil da edificação. } \\
\text { Considerar os sistemas construtivos assim como os efeitos de conforto. }\end{array}$ \\
\hline \multicolumn{2}{|r|}{ Categoria - Conservação de recursos materiais } \\
\hline $\begin{array}{l}\text { Qualidade dos materiais } \\
\text { e componentes }\end{array}$ & $\begin{array}{c}\text { Visa reduzir o consumo de recursos naturais utilizados para correção, por } \\
\text { meio do indicador de qualidade do PBQP-H evitando assim o uso de } \\
\text { produtos de baixa qualidade. }\end{array}$ \\
\hline $\begin{array}{l}\text { Componente } \\
\text { industrializado ou pré- } \\
\text { fabricado }\end{array}$ & $\begin{array}{l}\text { Empregar componentes industrializados com o intuito de reduzir a } \\
\text { geração de resíduos e perdas de materiais, assim como, o consumo de } \\
\text { recursos naturais. Adotar sistemas construtivos de componentes } \\
\text { industrializados montados em canteiro, projetados de acordo com as } \\
\text { normas ou no âmbito do Sistema Nacional de Aprovação Técnica } \\
\text { (SINAT) e emprega-los em dois dos itens: fachadas, divisórias internas, } \\
\text { estrutura de pisos (lajes) e escadas, pilares e vigas. }\end{array}$ \\
\hline $\begin{array}{l}\text { Fôrmas e escoras } \\
\text { reutilizáveis }\end{array}$ & $\begin{array}{c}\text { Visa reduzir a madeira em aplicações de baixa durabilidade, diminuindo o } \\
\text { desperdício e o impacto ambiental do uso de madeira, e aumentar o uso } \\
\text { de materiais recicláveis. Por meio de projetos de fôrmas, de acordo com a } \\
\text { NBR } 14931 \text { e especificação de uso. }\end{array}$ \\
\hline $\begin{array}{l}\text { Gestão de Resíduos de } \\
\text { Construção e Demolição } \\
\text { (RCD) }\end{array}$ & $\begin{array}{c}\text { Objetiva reduzir a quantidade de resíduos de construção e demolição, } \\
\text { assim como, seus impactos no meio ambiente urbano. A gestão do RCD } \\
\text { deve estar de acordo com as normas e avaliado pelo "Projeto de } \\
\text { Gerenciamento de Resíduos da Construção Civil - PGRCC" para cada } \\
\text { obra. }\end{array}$ \\
\hline \multicolumn{2}{|r|}{ Categoria - Práticas sociais } \\
\hline $\begin{array}{c}\text { Educação para gestão de } \\
\text { RCD }\end{array}$ & $\begin{array}{l}\text { Este faz parte do plano de gestão de RCD, com intuito de instruir os } \\
\text { agentes do processo de desenvolvimento do edifício para que tenham as } \\
\text { competências necessárias para a execução da obra. }\end{array}$ \\
\hline $\begin{array}{l}\text { Orientação aos } \\
\text { moradores }\end{array}$ & $\begin{array}{l}\text { Instruir os moradores quanto a forma de uso e manutenção da edificação, } \\
\text { com objetivo de maximizar o seu tempo de vida útil da, por meio de } \\
\text { informativos, manuais ilustrativos e didáticos. }\end{array}$ \\
\hline
\end{tabular}

Fonte: John e Prado (2010)

\section{LEED}

As categorias com abordagem de ACV do sistema LEED são Uso racional da água; Energia e atmosfera; Materiais e recursos; e a categoria Inovação e processo do projeto.

\section{Quadro 4 - Categorias com abordagem de ACV do LEED}

\begin{tabular}{|c|c|}
\hline Critério & Descrição \\
\hline \multicolumn{2}{|c|}{ Categoria - Uso Racional da Água } \\
\hline \begin{tabular}{c} 
Uso racional básico para casas \\
Categoria - Energia e Atmosfera \\
\hline $\begin{array}{c}\text { Desempenho Energético } \\
\text { Aprimorado }\end{array}$ \\
demanda por água.
\end{tabular} \\
\hline
\end{tabular}




\begin{tabular}{|c|c|}
\hline $\begin{array}{c}\text { Gerenciamento do Gás } \\
\text { Refrigerante }\end{array}$ & $\begin{array}{l}\text { Seleção de equipamentos de refrigeração das habitações de forma } \\
\text { eficiente. }\end{array}$ \\
\hline \multicolumn{2}{|c|}{ Categoria - Materiais e Recursos } \\
\hline $\begin{array}{c}\text { Gerenciamento de Resíduos } \\
\text { da Construção }\end{array}$ & $\begin{array}{l}\text { Visa a criação de planos para gerenciar os resíduos provenientes da } \\
\text { construção e operação. }\end{array}$ \\
\hline $\begin{array}{c}\text { Materiais ambientalmente } \\
\text { preferíveis }\end{array}$ & $\begin{array}{c}\text { Visa a utilização de materiais que sejam provenientes de reuso, } \\
\text { regionais, de conteúdo reciclado, de rápida renovação ou recicláveis. }\end{array}$ \\
\hline $\begin{array}{c}\text { Materiais Certificados } \\
\text { Escolha de materiais que receberam certificados nacionais ou } \\
\text { internacionais, conforme as normas: NBR ISO 14020:2002; Tipo I - } \\
\text { NBR ISO 14024: 2004; Tipo II - NBR ISO 14021: 2004; Tipo III - } \\
\text { NBR ISO 14025: 2006. }\end{array}$ \\
\hline $\begin{array}{c}\text { Desmontabilidade e Redução } \\
\text { de Resíduo }\end{array}$ & $\begin{array}{c}\text { Visa a escolha de sistemas estruturais e elementos não estruturais de } \\
\text { fácil desmontabilidade na fase de desativação. }\end{array}$ \\
\hline \multicolumn{2}{|c|}{ Categoria - Inovação e Processo do Projeto } \\
\hline Gerenciamento da Qualidade & \multicolumn{1}{c|}{ Visa durabilidade da edificação em geral. } \\
\hline \multicolumn{2}{|c|}{$\begin{array}{c}\text { Análise de Ciclo de Vida } \\
\text { certificada com base na norma NBR ISO 14040. }\end{array}$} \\
\hline
\end{tabular}

Fonte: USGBC (2009)

\subsection{Identificação das fases de ciclo de vida da edificação utilizadas nos sistemas}

O ciclo de vida de uma edificação é constituído por processo informativo e físico. No processo físico estão as fases de planejamento e projeto. O processo físico é constituído pelas fases: extração, transporte, fabricação dos materiais, construção, uso e operação, manutenção e desativação. O Quadro 5 indica as fases utilizadas nos processos de CAE.

\section{Quadro 5 - Fases do ciclo de vida pertencentes a cada CAE}

\begin{tabular}{|c|c|c|c|c|}
\cline { 2 - 5 } \multicolumn{1}{c|}{} & \multicolumn{4}{c|}{ Certificações Ambientais } \\
\hline Fases de processo (Físico) & AQUA & BREEAM & Casa Azul & LEED \\
\hline Extração & & $\mathrm{X}$ & $\mathrm{X}$ \\
\hline Transporte & $\mathrm{X}$ & $\mathrm{X}$ & $\mathrm{X}$ & $\mathrm{X}$ \\
\hline Fabricação de materiais & $\mathrm{X}$ & $\mathrm{X}$ & $\mathrm{X}$ & $\mathrm{X}$ \\
\hline Construção & $\mathrm{X}$ & $\mathrm{X}$ & $\mathrm{X}$ & $\mathrm{X}$ \\
\hline Uso & & $\mathrm{X}$ & $\mathrm{X}$ & $\mathrm{X}$ \\
\hline Manutenção & $\mathrm{X}$ & $\mathrm{X}$ & & $\mathrm{X}$ \\
\hline Desativação & $\mathrm{X}$ & & & $\mathrm{X}$ \\
\hline
\end{tabular}

Fonte: Autores

\subsection{Correlação dos aspectos e impactos dos principais métodos de caracterização da fase de AICV (Avaliação de Impacto do Ciclo de Vida) com as categorias de impacto dos sistemas selecionados}

Os métodos para a classificação das categorias de avaliação de impacto de ciclo de vida das certificações ambientais escolhidos para este estudo são os mais utilizados atualmente, Ecoindicator99 (2001), CML2001, EPS2000, LIME (2003), EDIP97, IMPACT2002+. O Quadro 6 mostra a relação as categorias de impacto dos métodos de AICV que são utilizadas nas categorias de avaliação das CAE.

No Quadro 6 indica-se que as categorias de Material Particulado, Radiação Ionizante, Ecotoxicidade, Acidificação, Eutrofização não são avaliadas no processo das CAE. As 
categorias de impacto abordadas por todas as CAE são as Toxicidade Humana, Mudança Climática, Destruição da Camada de Ozônio, Extração ou uso de combustíveis fósseis ou não renováveis, Uso da água, Mudança em valores de recreação.

Quadro 6 - Categorias de impacto dos métodos de AICV abordadas pelos CAE

\begin{tabular}{|c|c|c|c|c|c|}
\hline $\begin{array}{l}\text { Área de } \\
\text { proteção }\end{array}$ & Categorias de Impacto & AQUA & BREEAM & Casa Azul & LEED \\
\hline \multirow{11}{*}{ 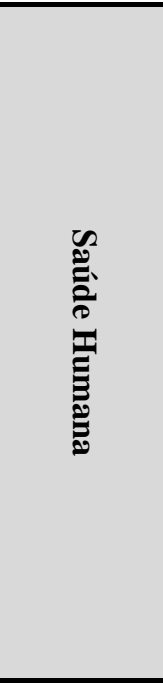 } & $\begin{array}{c}\text { Incômodo severo, Incômodo (doença } \\
\text { ligeira) }\end{array}$ & $\mathrm{X}$ & - & - & - \\
\hline & Toxicidade Humana & $\mathrm{X}$ & $\mathrm{X}$ & $\mathrm{X}$ & $\mathrm{X}$ \\
\hline & Contaminação Atmosférica Urbana & $\mathrm{X}$ & - & - & $\mathrm{X}$ \\
\hline & Efeitos Respiratórios & $\mathrm{X}$ & $\mathrm{X}$ & - & - \\
\hline & Material Particulado & - & - & - & - \\
\hline & Mudança Climática & $\mathrm{X}$ & $\mathrm{X}$ & $\mathrm{X}$ & $\mathrm{X}$ \\
\hline & Destruição da Camada de Ozônio & $\mathrm{X}$ & $\mathrm{X}$ & $\mathrm{X}$ & $\mathrm{X}$ \\
\hline & Oxidação Fotoquímica & $\mathrm{X}$ & $\mathrm{X}$ & - & - \\
\hline & Radiação Ionizante & - & - & - & - \\
\hline & Ruído & - & $\mathrm{X}$ & - & $\mathrm{X}$ \\
\hline & Odor (malcheiroso) água, ar & $\mathrm{X}$ & - & - & - \\
\hline \multirow{5}{*}{ 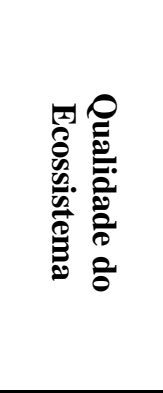 } & Ecotoxicidade & - & - & - & - \\
\hline & Acidificação & - & - & - & - \\
\hline & Eutrofização & - & - & - & - \\
\hline & Uso e Ocupação do Solo & - & $\mathrm{X}$ & $\mathrm{X}$ & $\mathrm{X}$ \\
\hline & $\begin{array}{c}\text { Dessecação (grupo de problemas } \\
\text { ambientais relacionados a falta de água } \\
\text { devido a extração de água subterrânea e } \\
\text { a provisão de água potável) }\end{array}$ & - & $\mathrm{X}$ & - & $\mathrm{X}$ \\
\hline \multirow{4}{*}{ 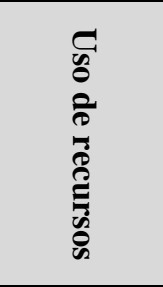 } & $\begin{array}{c}\text { Diminuição de Recursos (abióticos) e } \\
\text { (bióticos) }\end{array}$ & - & $\mathrm{X}$ & $\mathrm{X}$ & - \\
\hline & Extração de Minerais & - & $\mathrm{X}$ & - & $\mathrm{X}$ \\
\hline & $\begin{array}{c}\text { Extração ou uso de combustíveis fósseis } \\
\text { ou não renováveis } \\
\end{array}$ & $\mathrm{X}$ & $\mathrm{X}$ & $\mathrm{X}$ & $\mathrm{X}$ \\
\hline & Uso da água & $\mathrm{X}$ & $\mathrm{X}$ & $\mathrm{X}$ & $\mathrm{X}$ \\
\hline Resíduos & Resíduos & $\mathrm{X}$ & $\mathrm{X}$ & $\mathrm{X}$ & - \\
\hline Cultura & Mudança em valores de recreação & $\mathrm{X}$ & $\mathrm{X}$ & $\mathrm{X}$ & $\mathrm{X}$ \\
\hline Acidentes & Acidentes & - & - & - & - \\
\hline Calor & Liberação de Calor & - & - & - & $\bar{X}$ \\
\hline
\end{tabular}

Fonte: Autores

A AQUA avalia categorias que outas CAE não avaliam, como a categoria de Incômodo severo e Odor. A certificação LEED é a única dentre elas que avalia a categoria Liberação de Calor.

Após essa análise de categorias foi relacionado a quantidade de categorias de impacto utilizadas pelas CAE com cada método de AICV na Tabela 1.

A Tabela 1 indica que as categorias do método EPS são mais utilizadas por todas as CAE. A AQUA possui menos categorias do Eco-Indicator e do EDIP. As certificações BREEAM, Casa Azul e LEED têm poucas categorias do método EDIP. 
Tabela 1 - Relação das CAE com os métodos de AICV

\begin{tabular}{|c|cccc|}
\hline Métodos de Caracterização da AICV & \multicolumn{4}{c}{ Certificações Ambientais } \\
Eco-Indicator 99 & AQUA & BREEAM & Casa Azul & LEED \\
CML2001 & 3 & 5 & 3 & 4 \\
EPS2000 & 4 & 7 & 4 & 5 \\
LIME & 7 & 10 & 7 & 7 \\
EDIP97 & 5 & 5 & 5 & 4 \\
\hline Impact2002+ & 3 & 3 & 2 & 2 \\
\hline
\end{tabular}

Fonte: Autores

\section{CONSIDERAÇÕES FINAIS}

Os resultados até esta etapa da pesquisa nos permite fazer algumas considerações, quanto aos materiais, componentes e sistemas construtivos são avaliados por seus impactos nas categorias de extração de recursos, energia incorporada, conteúdo de material reaproveitado, emissões de gases do efeito estufa ou destruidores da camada de ozônio e pela categoria de qualidade do ambiente interno.

As questões das fases do ciclo de vida da edificação nos sistemas de avaliação ambiental de edificações mostram que as CAE não avaliam todos os processos de forma conjunta e dinâmica, o processo é realizado por fases isoladas. Em alguns casos há uma preocupação com o ciclo de vida do edifício como um todo, como nas áreas de proteção relacionadas à saúde humana e uso de recursos. No entanto, trata-se de avaliações qualitativas, para as quais não há uma ferramenta técnica que avalie os impactos potenciais de forma a quantificar o ciclo.

Em relação aos métodos de AICV, a análise mostrou que todas as CAE utilizam as categorias de avaliação de impacto do método EPS em suas certificações. O método ECO-Indicator e o EDIP são categorias que os sistemas CAE pouco utilizam.

Tanto os sistemas de CAE quanto a técnica de ACV tem o objetivo de criar um ciclo de vida sustentável para o ambiente construído. Embora tenham metodologias diferentes, qualitativa e quantitativa respectivamente, ambos possuem pontos fortes e fracos e devem preferencialmente ser utilizadas em conjunto. Para facilitar a utilização da ACV em CAE é necessário que os critérios das certificações sejam além de classificações por critérios de categorias e tenham banco de dados público dos produtos a serem utilizados na edificação.

\section{AGRADECIMENTOS}

À CAPES, pelo apoio recebido.

\section{REFERÊNCIAS}

ASSOCIAÇÃO BRASILEIRA DE NORMAS TÉCNICAS (ABNT). NBR ISO 14031: Gestão ambiental - Avaliação de desempenho ambiental - Diretrizes. Rio de Janeiro, 2004.

NBR ISO 14040: Gestão ambiental - Avaliação do ciclo de vida - Princípios e estrutura. Rio de Janeiro, 2009. 
BUILDING RESEARCH ESTABLISHMENT, BRE. Building Research Establishment Environmental Assessment Method Centre. (2009). Code for sustainable homes technical guidance. Disponível em: $\langle$ http://www.breeam.org $>$. Acesso em: 18 set. 2013.

BUENO, C. (2010). Desempenho ambiental de edificações: cenário atual e perspectivas dos sistemas de certificação. Revista Minerva, v.7, n.1, p.45-52.

COLE, R. J. (1998). Charting the future: emerging trends in building environmental assessment methods, School of Architecture, University of British Columbia. Building Research and Information, v.26, n.1, p.3-16.

FUNDAÇÃO CARLOS ALBERTO VANZOLIN , FCAV. (2014). Referencial de avaliação da qualidade ambiental de edifícios residenciais em construção processo AQUA: vs.1. Disponível em: < http://vanzolini.org.br/download/RT_AQUAHQE-Edificios_residenciais.pdf>. Acesso em: 1 ago. 2014.

JOHN, V.M.; PRADO, R.T.A. (Coord.). (2010). Boas práticas para habitação mais sustentável - selo CASA AZUL. São Paulo: Páginas e Letras.

KLEIN, S. E. S.; SELL, I. (2002). Gestão Ambiental na Construção Civil de Edificações. In: VII ENGEMA - ENCONTRO NACIONAL DE GESTÃO EMPRESARIAL E MEIO AMBIENTE, São Paulo, 2002.

REBITZER, G. et al. (2004). Life cycle assessment. Part 1: Framework, goal and scope definition, inventory analysis, and applications. Environment International, v.30, p.701-720.

SEVERO, E. M. F.; CARVALHO FILHO, A. C.; SOUSA, H. J. C. (2012). Comparativo das principais ferramentas para avaliação do ciclo de vida de edificações. In: CONGRESSO BRASILEIRO EM GESTÃO DO CICLO DE VIDA DE PRODUTOS E SERVIÇOS, 3., 2012, Maringá. Anais... [S.1.:s.n.].

SILVA, V. G. (2003). Avaliação da sustentabilidade de edifícios de escritórios brasileiros: diretrizes e base metodológica. Tese (Doutorado) - Universidade de São Paulo, São Carlos, 2003.

SILVA, G. A. (2007). Avaliação do ciclo de vida - sua importância para a sustentabilidade da construção civil. In: CICLO DE DEBATES SOBRE CONSTRUÇÃO CIVIL SUSTENTÁVEL "MEIO AMBIENTE EM DEBATE" Insumos e Resíduos. São Paulo. Disponível em: <http://www.ambiente.sp.gov.br /wpcontent/uploads/noticias/2007/10/gil.pdf>. Acesso em: 10 ago. 2014.

SOCIETY OF ENVIRONMENTAL TOXICOLOGY AND CHEMISTRY. SETAC Foundation for Environmental Education. (1991). A Technical framework for life-cycle assessment, Washington, DC: Society of Environmental Toxicology and Chemistry and SETAC Foundation for Environmental Education Inc. In: WORKSHOP HELD IN SMUGGLERS NOTCH, 1990, Vermont.

UNITED STATES GREEN BUILDING COUNCIL, USGBC. (2009). Green building rating system: LEED for new construction and major renovation - with alternative compliance paths for projects outside the U.S. [S.1.:s.n.].

ZAMBRANO, L. M. de A.; BASTOS, L. E. G.; SLAMA, J. G. (2004) Gestão Ambiental e Avaliação do desempenho da edificação - estudo de caso na indústria farmacêutica. In: I CONFERÊNCIA LATINO-AMERICANA DE CONSTRUÇÃO SUSTENTÁVEL. X ENCONTRO NACIONAL DE TECNOLOGIA DO AMBIENTE CONSTRUÍDO (ENTAC). 2004, São Paulo. Anais... São Paulo, ISBN 85-89478-08-4. 